\title{
NON-LOCAL ELECTRON-POSITRON ENHANCEMENT FACTORS IN SOLIDS
}

\author{
A. RubaszeK* \\ W. Trzebiatowski Institute of Low Temperature and Structure Research \\ Polish Academy of Sciences, P.O. Box 937, 50-950 Wrocław 2, Poland \\ Z. SzoteK ${ }^{\dagger}$ and W.M. Temmerman ${ }^{\ddagger}$ \\ Daresbury Laboratory, Warrington, WA4 4AD, Cheshire, U.K.
}

Non-local electron-positron correlation effects in solids are studied. The weighted density approximation is applied to calculations of the non-local electron-positron correlation functions. The calculated weighted density approximation electron-positron enhancement factors for the core electrons are compared with those obtained within the local density approximation. Also, differences in the electron-positron enhancement factors due to the $s, p, d$ and $f$ angular momentum channels of the electron charge density are studied. The formalism is applied to $a b$ initio calculations of positron lifetimes in a variety of metals and silicon. The influence of various approximations to the electron-positron interaction on the positron lifetimes is also presented. The weighted density approximation results are compared to those calculated within the local density approximation, the recent generalized gradient approximation and with experimental data.

PACS numbers: 78.70.Bj, 71.10.-w, 71.60.tz

The positron lifetime, $\tau$, is an important characteristic of electronic properties of solids. This parameter provides information on the electron density distribution in the host material, thus yielding also useful information on defects in metals and semiconductors. The positron annihilation rate, $\lambda=1 / \tau$, is calculated as

$$
\lambda=\pi r_{0}^{2} c \int n_{\mathrm{el}}\left(r_{\mathrm{p}}\right)\left|\psi_{+}\left(r_{\mathrm{p}}\right)\right|^{2} g\left(r_{\mathrm{p}}, r_{\mathrm{p}}\right) \mathrm{d} r_{\mathrm{p}},
$$

where $n_{\mathrm{el}}\left(r_{\mathrm{e}}\right)$ is the electron density in the host material, $\psi_{+}\left(r_{\mathrm{p}}\right)$ is the wave function of a thermalised positron and $g\left(r_{\mathrm{e}}, r_{\mathrm{p}}\right)$ denotes the correlation function of the positron at $r_{\mathrm{p}}$ and electrons at $r_{\mathrm{e}}$, and $r_{0}$ and $c$ are the classical electron radius and velocity of light, respectively.

*e-mail: ania@highscreen.int.pan.wroc.pl

†e-mail: Z.Szotek@dl.ac.uk

†e-mail: W.M.Temmerman@dl.ac.uk 
The electron charge density, $n_{\mathrm{el}}\left(r_{\mathrm{e}}\right)$, at the position $r_{\mathrm{e}}$ in the host material, consists of a core, $n_{c}$, and valence, $n_{\mathrm{v}}$, contribution. The valence contribution, $n_{\mathrm{v}}\left(r_{\mathrm{e}}\right)$, can be further decomposed into the angular momentum components, $n_{l}\left(r_{e}\right)$, of which we shall make use in the calculations of the electron-positron (e-p). correlation functions. Consequently, throughout this paper, we shall use quantities with a subscript $t$, when considering specific contributions due to different types of electrons. Here $t$ may stand for: c (core), $\mathrm{v}$ (valence), $s, p, d$, and $f$ electrons. In this notation we can write

$$
n_{\mathrm{el}}\left(r_{\mathrm{p}}\right) g\left(r_{\mathrm{p}}, r_{\mathrm{p}}\right)=\sum_{t} n_{t}\left(r_{\mathrm{p}}\right) g_{t}\left(r_{\mathrm{p}}, r_{\mathrm{p}}\right)
$$

It has been a common practice in calculations of the positron lifetimes to treat the position-dependent $\mathrm{e}-\mathrm{p}$ interaction within the local density approximation (LDA) [1-7]. In this approximation the e-p correlations are replaced by their analogues in a homogeneous electron gas with the local electron density $n\left(r_{\mathrm{p}}\right)$ at the positron position $r_{\mathrm{p}}$. This approximation is known to work well for systems with slowly varying electron densities, e.g. for valence electrons in simple metals. However, for core electrons, due to strong variations of the density, the LDA is not expected to work very well, while theoretical [4-6, 8-10], semi-empirical [11], and experimental [12] studies have shown that the core electrons contribution to the annihilation characteristics is non-negligible in comparison with the valence electrons contribution. For the localized core electrons the LDA seems to overestimate the e-p correlation effects, and in particular, in the interstitial region. The same occurs for localized $d$ and $f$ electrons in transition metals, rare earths and actinides. As a result, the LDA underestimates the positron lifetimes, as compared with the experimental data [13]. This discrepancy may be caused by non-local effects.

A certain form of non-locality of the e-p interaction was introduced in Refs. $[10,14,15]$, by evaluating the e-p correlation function $g\left(r_{\mathrm{p}}, r_{\mathrm{p}}\right)$ at the positron position within the generalized gradient approximation (GGA). This approach, however, makes use of a parameter-dependent $\mathrm{e}-\mathrm{p}$ correlation function and the adjustable parameter is fitted to the experimental data.

In this paper we utilize the weighted density approximation (WDA) to calculate the non-local e-p correlation functions in solids [8]. The weighted density approximation includes non-local effects through substituting the electron density, $n\left(r_{\mathrm{p}}\right)$, in the LDA correlation function, by its weighted average, $n^{*}\left(r_{\mathrm{p}}\right)$, where the distribution of the electron polarization cloud, surrounding positron, provides the weighting factor. The WDA was first introduced by Gunnarsson et al. [16] for studying the non-local electron-electron correlations and exchange effects. The WDA can be interpreted as an $a b$ initio generalization of the LDA for strongly inhomogeneous systems. When the electron density is slowly varying, the WDA reduces to the LDA. The former has been successfully applied to the problem of positron interaction with a metal surface $[17,18]$, leading to a much improved description of the positron screening at surfaces, as compared with the LDA.

In the non-local approach of the WDA $[8,17,18]$, the correlation functions, $g_{t}^{\mathrm{WDA}}\left(r_{\mathrm{e}}, r_{\mathrm{p}}\right)$ are approximated by their analogues in an electron gas, $g_{t}^{h}\left[\left|r_{\mathrm{e}}-r_{\mathrm{p}}\right|, n_{t}^{*}\left(r_{\mathrm{p}}\right)\right]$, where $n_{t}^{*}\left(r_{\mathrm{p}}\right)$ is an effective. WDA density for electrons of 
type $t$. The density $n_{t}^{*}\left(r_{\mathrm{p}}\right)$ is defined, for any positron position $r_{\mathrm{p}}$ and any type of electrons $t$, as the solution of the charge neutrality condition [8] which states that the electron charge, screening a positron, is equal to $-e$.

$$
\begin{array}{r}
\int \mathrm{d} r_{\mathrm{e}} n_{t}\left(r_{\mathrm{e}}\right)\left\{g_{t}^{h}\left[\left|r_{\mathrm{e}}-r_{\mathrm{p}}\right|, n_{t}^{*}\left(r_{\mathrm{p}}\right)\right]-1\right\} \\
=\frac{\left\{g_{t}^{h}\left[0, n_{\mathrm{el}}\left(r_{\mathrm{p}}\right)\right]-1\right\} n_{t}\left(r_{\mathrm{p}}\right)}{\sum_{t^{\prime}}\left\{g_{t^{\prime}}^{h}\left[0, n_{\mathrm{el}}\left(r_{\mathrm{p}}\right)\right]-1\right\} n_{t^{\prime}}\left(r_{\mathrm{p}}\right)} .
\end{array}
$$

The corresponding annihilation rates for electrons of type $t$ are

$$
\lambda_{t}^{\mathrm{WDA}}=\int \mathrm{d} r_{\mathrm{p}} n_{t}\left(r_{\mathrm{p}}\right)\left|\psi_{+}\left(r_{\mathrm{p}}\right)\right|^{2} g_{t}^{h}\left[0, n_{t}^{*}\left(r_{\mathrm{p}}\right)\right]
$$

and the correlation functions $g_{t}^{h}(0, n)$ have been parameterized as a function of density $n$ in several works $[19,20]$. When $n_{t}^{*}\left(r_{\mathrm{p}}\right)$ is replaced by $n_{\mathrm{el}}\left(r_{\mathrm{p}}\right)$, Eq. (3) gives the LDA annihilation rate. Substituting $g_{t}\left(r_{\mathrm{p}}, r_{\mathrm{p}}\right)$ by unity we get the independent particles model (IPM) result.

In the present calculations, the self-consistent electron densities, $n_{\mathrm{el}}\left(r_{\mathrm{e}}\right)$ and $n_{t}\left(r_{\mathrm{e}}\right)$, have been obtained using the linear muffin tin orbitals (LMTO) method in the atomic sphere approximation (ASA) [21]. For the core electrons we have implemented the frozen core approximation. This does not seem to appreciably influence the non-local effects studied in the present paper. Indeed, the WDA effective density for the core electrons is much less dependent on the positron position than the core electron density in the host material. Therefore, small changes in the core electron density, due to core electron relaxation, are not expected to alter the WDA results.

To discuss the effect of the enhancement of the electron density at the positron position on the positron annihilation rates, it is convenient to define, following Refs. $[4,6 ; 1,10,14]$, the average enhancement factors $\Gamma_{t}^{\mathrm{LDA}}=\lambda_{t}^{\mathrm{LDA}} / \lambda_{t}^{\mathrm{IPM}}$ and $\Gamma_{t}^{\mathrm{WDA}}=\lambda_{t}^{\mathrm{WDA}} / \lambda_{t}^{\mathrm{IPM}}$, respectively for the LDA and WDA. These average enhancement factors $\Gamma_{t}$ can be interpreted as the enhancement factors $g_{t}\left(r_{\mathrm{p}}, r_{\mathrm{p}}\right)$ in the region, where the overlap of the various electron distributions with the positron distribution is the largest. The quantities $\Gamma_{t}$ are shown in Figs. 1-3, for a number of alkali metals, $3 d, 4 d$ and $5 d$ transition metals.

In all the systems we studied, the quantities $\Gamma_{\mathrm{c}}$ for the core electrons are larger than unity, indicating some deviation from the IPM. Moreover, except for $\mathrm{Li}$, $\mathrm{Al}$, and $\mathrm{Si}$, the WDA e-p enhancement factors are generally considerably smaller than the LDA values. In transition metals, the core electrons are distributed over the high density region. Therefore, the values of $\Gamma_{\mathrm{c}}^{\mathrm{LDA}(\mathrm{WDA})}$ are not too large (smaller than 2.3). As a consequence, the core electrons enhancement factors of the alkali metals are greater than the ones in the transition metals. It may be of interest that the values of $\Gamma_{\mathrm{c}}$, obtained within GGA by Alatalo et al. [10] for Al, $\mathrm{Si}, \mathrm{Ni}$, and $\mathrm{Cu}$, are very similar to the $\Gamma_{\mathrm{c}}^{\mathrm{WDA}}$ of the present calculation.

The $\Gamma_{s(p)}^{\mathrm{WDA}}$ 's and $\Gamma_{s(p)}^{\mathrm{LDA}}$ 's are very similar, with a trend of slightly larger $\Gamma_{s(p)}^{W D A}$ 's. In fact, there is no substantial difference between WDA and LDA for nearly-free $s$ and $p$ valence electrons. This is not the case for localized $d$ electrons in transition metals (see Figs. 2 and 3). The WDA enhancement factor for $d$ electrons 


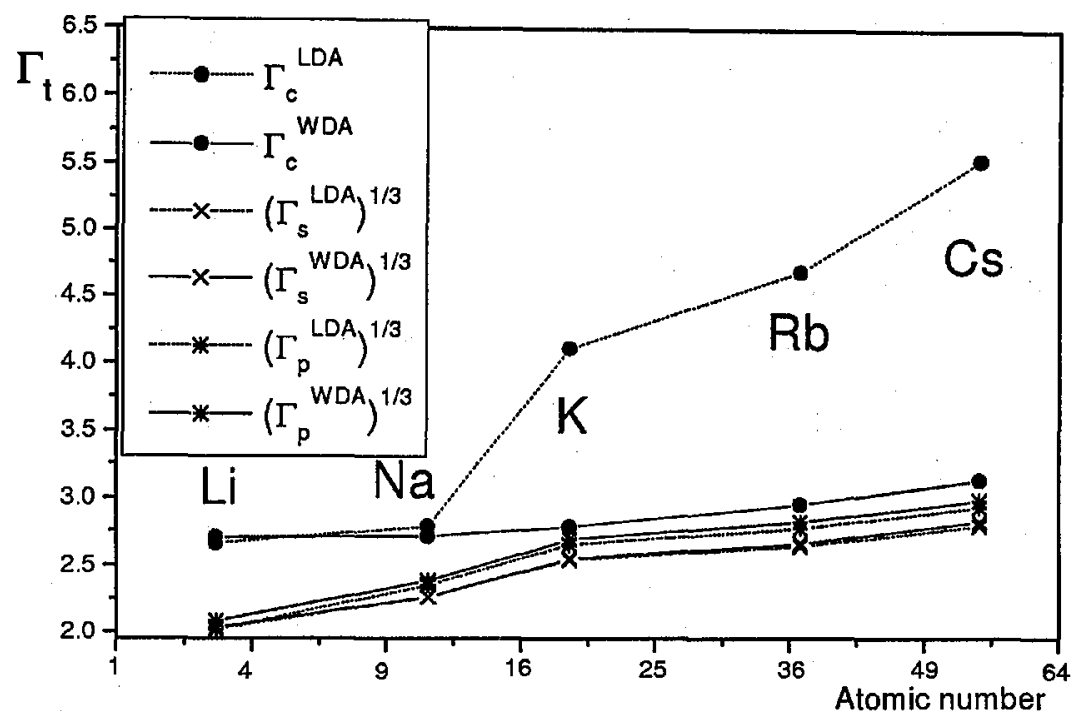

Fig. 1. Average LDA and WDA enhancement factors $\Gamma_{t}$ for various types of electrons $t$ in alkali metals.

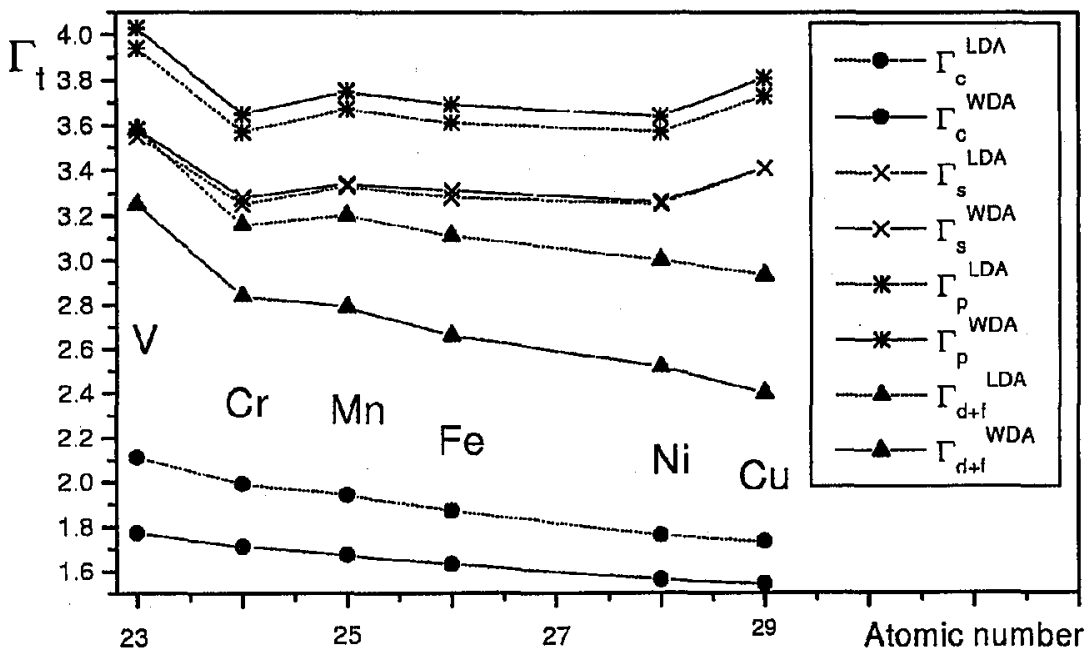

Fig. 2. Average LDA and WDA enhancement factors $\Gamma_{t}$ for various types of electrons $t$ in $3 d$ transition metals.

is strongly reduced as compared with its LDA counterpart. The reason is that the $d$ electrons' contribution to the polarization cloud, screening the positron, is more likely found in the region where the $d$ electrons reside, than it would follow from the LDA. As a result, the $d$ part of the screening charge is shifted from the interstitial region (where the positron is found with the highest probability) towards the intermediate region. Thus, the overlap of positron density with electrons of type $d$ is diminished in comparison with the LDA. 


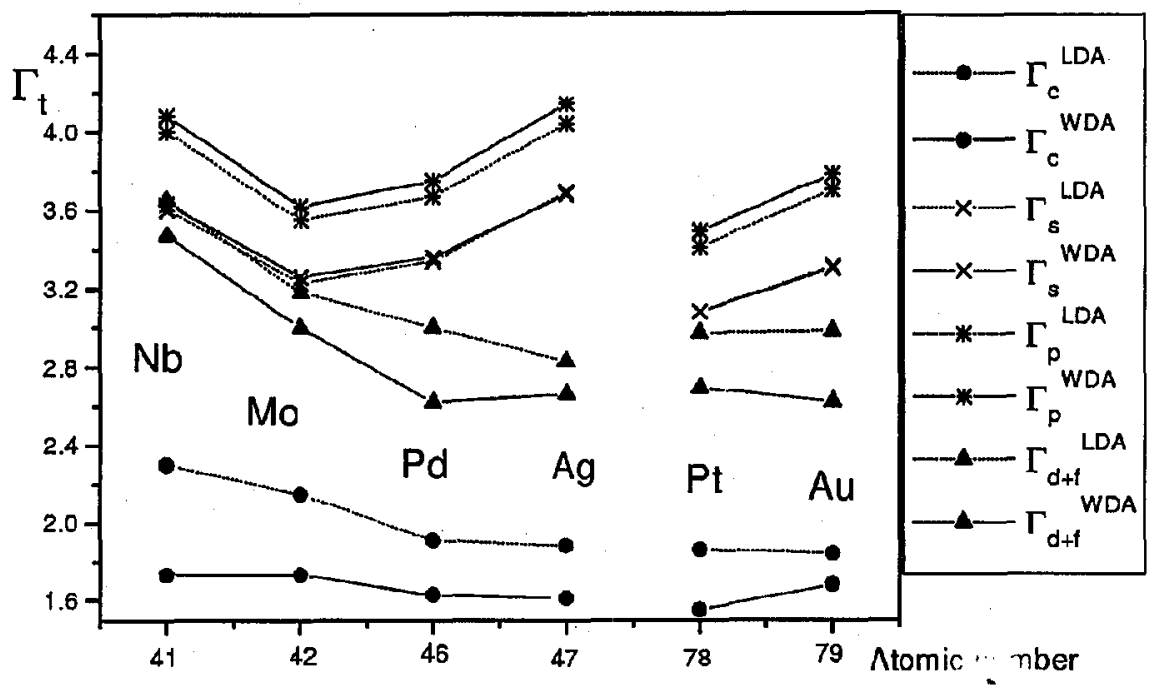

Fig. 3. Average LDA and WDA enhancement factors $\Gamma_{t}$ for various types of electrons $t$ in $4 d, 5 d$ transition metals.

Calculated positron lifetimes, obtained within LDA and WDA, are compared in Table with the measured values [13], and with the GGA calculation of Ref. [14]. In the last row of Table, we give the mean difference between the calculated and experimental positron lifetimes, namely, error $=(1 / n) \sum_{i}\left|\tau_{i}^{\text {cal }}-\tau_{i}^{\text {exp }}\right|$, a veraged over the systems listed in Table. These errors provide a quantitative assessment of the WDA and LDA results with respect to the experimental data.

When analysing the values quoted in the last row of Table one can see that the WDA gives the best results. Note that also the state-selective LDA results (Eq. (3)) for the systems listed in Table are in better agreement with the experiment than the GGA values [14] (for more details concerning the state-selective LDA approach see Refs. $[1,8])$.

Summarizing the results of this paper it is fair to say that the WDA gives a substantially different picture of the e-p correlations in solids than the LDA. Comparing the WDA with the LDA, we find, as expected, that for nearly-free electron like systems both approaches give similar annihilation rates. However, non-local effects are very important for the core electrons contribution to the total annihilation rates. They are equally important for the $d$ electrons in transition metals. These non-local effects are included in the WDA in an average manner, through distributing the partial electron densities over the whole Wigner-Seitz cell. Comparing the present theory and the GGA approach [14] one should note that the WDA enhancement factors for nearly-free electrons differ appreciably from the corresponding quantities for the localized core and $d$ electrons, whilst within GGA [14] all types of electrons scatter on the positron at the same rate. Therefore, although the WDA and GGA $[10,14]$ provide similar patterns in the enhancement factors for the core electrons, as compared with the LDA, nevertheless, considerable differences are observed between the WDA and GGA enhancement factors for valence electrons, both in simple and transition metals. 


\section{TABLE}

Positron lifetimes calculated using the LDA and WDA compared with the GGA calculation and the experimental lifetimes of Ref. [13(a)]. The experimental value of Ref. [13(b)] is quoted in parentheses.

\begin{tabular}{|c|c|c|c|c|}
\hline Element & LDA & WDA & GGA & Experiment \\
\hline $\mathrm{Al}$ & 165.9 & 163.3 & 153 & 163 \\
\hline $\mathrm{Na}$ & 339.5 & 334.7 & 329 & 338 \\
\hline $\mathrm{K}$ & 383.2 & 395.5 & 392 & 397 \\
\hline $\mathrm{V}$ & 118.4 & 129.8 & 119 & 130 \\
\hline $\mathrm{Fe}$ & 102.1 & 111.8 & 108 & 106 \\
\hline $\mathrm{Ni}$ & 97.3 & 112.1 & 107 & 110 \\
\hline $\mathrm{Cu}$ & 106.6 & 122.8 & 118 & $110(121)$ \\
\hline $\mathrm{Nb}$ & 124.3 & 129.4 & 122 & 119 \\
\hline Mo & 105.5 & 109.7 & 112 & 103 \\
\hline $\mathrm{Pd}$ & 103.9 & 114.9 & 114 & 96 \\
\hline $\mathrm{Pt}$ & 95.1 & 103.1 & 101 & 99 \\
\hline $\mathrm{Si}$ & 211.7 & 217.0 & 210 & 219 \\
\hline \multicolumn{5}{|c|}{ Mean error for 12 elements } \\
\hline & 6.39 & 5.68 & 7.42 & - \\
\hline
\end{tabular}

In $\mathrm{Si}$, the WDA provides a more reliable description of the e-p correlations than the LDA. Therefore, this gives us confidence to apply the WDA in the calculation of $\mathrm{e}-\mathrm{p}$ momentum distributions, especially for Si. It is with $\mathrm{Si}$ and transition metals in mind that the present implementation has been undertaken in the first place.

We are grateful to the Royal Society for partial financial support of this work.

\section{References}

[1] S. Daniuk, M. Šob, A. Rubaszek, Phys. Rev. B 43, 2580 (1991).

[2] R.M. Nieminen, in: Positron Solid State Physics, Eds. W. Brandt, A. Dupasquier, North-Holland, Amsterdam 1983; E. Boroński, R.M. Nieminen, Phys. Rev. B 34, 3820 (1986).

[3] R.M. Nieminen, M.J. Puska, Phys. Rev. Lett. 50, 281 (1983); R.M. Nieminen, M.J. Puska, M. Manninen, ibid. 53, 1298 (1984); M.J. Puska, R.M. Nieminen, J. Phys. F 13, 2695 (1983).

[4] K.O. Jensen, J. Phys., Condens. Matter 1, 10595 (1989).

[5] P.A. Sterne, J.H. Kaiser, Phys. Rev. B 43, 13892 (1991).

[6] M.J. Puska, J. Phys., Condens. Matter 3, 3455 (1991); F. Plazaola, A.P. Seitsonen, M.J. Puska, ibid. 6, 8809 (1994). 
[7] B. Barbiellini, P. Genoud, T. Jarlborg, J. Phys., Condens. Matter 3, 7631 (1991).

[8] A. Rubaszek, Z. Szotek, W.M. Temmerman, Phys. Rev. B 58, 11285 (1998).

[9] B. Chakraborty, Phys. Rev. B 24, 7423 (1981); S. Daniuk, G. Kontrym-Sznajd, J. Majsnerowski, M. Šb, H. Stachowiak, J. Phys., Condens. Matter 1, 632 (1989).

[10] M. Alatalo, B. Barbiellini, M. Hakala, H. Kauppinen, T. Korhonen, M.J. Puska, K. Saarinen, P. Hautojärvi, R.M. Nieminen, Phys. Rev. B 51, 4776 (1995); ibid. 54, 2397 (1996).

[11] M. Sob, J. Phys. F 12, 571 (1982); O. Johnson, Phys. Status Solidi B 99, 745 (1980).

[12] A.H. Weiss, R. Mayer, M. Jibaly, C. Lei, D. Mehl, K.G. Lynn, Phys. Rev. Lett. 61, 2245 (1988); K.O. Jensen, A.H. Weiss, Phys. Rev. B 41, 3928 (1990); L. Oberli, A.A. Manuel, R. Sachot, P. Descout, M.J. Peter, ibid. 31, 6104 (1985).

[13] (a) A. Seeger, F. Banhart, W. Brauer, in: Positron Annihilation, Eds. L. Dorikens-Vanpraet, M. Dorikens, D. Segers, World Scientific, Singapore 1989, p. 275;

(b) N. Djourelov, M. Misheva, J. Phys., Condens. Matter 8, 2081 (1996).

[14] B. Barbiellini, M.J. Puska, T. Torsti, R.M. Nieminen, Phys. Rev. B 51, 7341 (1995); B. Barbiellini, M.J. Puska, T. Korhonen, A. Harju, T. Torsti, R.M. Nieminen, ibid. 53, 16201 (1996).

[15] B. Barbiellini, M. Hakala, M.J. Puska, R.M. Nieminen, Phys. Rev. B 56, 7136 (1997).

[16] O. Gunnarsson, M. Jonson, B.I. Lundqvist, Phys. Rev. B 20, 3136 (1979); R.O. Jones, O. Gunnarsson, Rev. Mod. Phys. 61, 689 (1989).

[17] A. Rubaszek, Phys. Rev. B 44, 10857 (1991); A. Rubaszek, A. Kiejna, S. Daniuk, J. Phys., Condens. Matter 5, 8195 (1993).

[18] K.O. Jensen, A.B. Walker, J. Phys. F 18, L277 (1988).

[19] S. Kahana, Phys. Rev. 129, 1622 (1963); W. Brandt, J. Reinheimer, Phys. Lett. A 35, 109 (1971); J. Arponen, E. Pajanne, J. Phys. C 12, 3013 (1979); J. Arponen, E. Pajanne, J. Phys. F 9, 2359 (1979); A. Kallio, P. Pietiläinen, L. Lantto, Phys. Scr. 25, 943 (1982); H. Stachowiak, Phys. Rev. B 41, 12522 (1990).

[20] A. Rubaszek, H. Stachowiak, Phys. Status Solidi B 124, 159 (1984); Phys. Rev. $B$ 38, 3846 (1988); A. Rubaszek, H. Stachowiak, E. Boroniski, Z. Szotek, ibid. 30, 2490 (1984).

[21] O.K. Andersen, Phys. Rev. B 12, 3060 (1975); W.R.L. Lambrecht, O.K. Andersen, ibid. 34, 2439 (1986). 